\title{
Evidence of Deconfinement and Search for the QCD Critical Point
}

\section{P. Seyboth for the NA49 collaboration ${ }^{* \dagger}$}

Max-Planck-Institut für Physik, Munich, Germany and Jan Kochanowski University, Kielce, Poland

E-mail: pxs@mppmu.mpg.de

The NA49 experiment obtained evidence for the onset of deconfinement from a study of hadron production in central $\mathrm{Pb}+\mathrm{Pb}$ collsisons at the CERN SPS. No indications were found yet for the critical point of QCD in measurements of fluctuations. Future plans for the continuation of the search are mentioned.

8th Conference Quark Confinement and the Hadron Spectrum

September 1-62008

Mainz, Germany

\footnotetext{
* Speaker.

$\dagger$ I am grateful for the invitation to show these NA49 results. Many thanks to all the NA49 collaborators who produced the presented measurements.
} 


\section{Introduction}

The study of nucleus-nucleus collisions at the CERN SPS offered for the first time the possibility of reaching energy densities in excess of $\approx 1 \mathrm{GeV} / \mathrm{fm}^{3}$ during the early stage of the reaction. Under these conditions QCD predicts a phase transition between hadron gas and a state of quasifree quarks and gluons, the quark gluon plasma (QGP). Indeed, predicted signatures of the QGP, e.g. strangeness enhancement, charmonia suppression and dilepton enhancement were observed in $\mathrm{Pb}+\mathrm{Pb}$ collisions at the top SPS energy [1]. Subsequent measurements of the energy dependence of various hadron production properties showed anomalies at low SPS energies which indicate that the onset of deconfinement occurs around $30 \mathrm{~A} \mathrm{GeV}$ beam energy [2]. Moreover, QCD suggests that the first order phase transition boundary between QGP and hadrons at high baryon density ends in a critical point and then turns into a rapid crossover at low baryon density [3]. This paper discusses experimental results from the NA49 experiment which are relevant for these topics.

The NA49 detector is a large acceptance spectrometer [4] employing a system of time projection chambers (TPCs) for efficient tracking in the forward hemisphere of the reactions, precise momentum reconstruction and particle identification using the energy loss $d E / d x$ in the TPC gas. Two time of flight (TOF) walls of 800 scintillator tiles each augment particle identification mainly near midrapidity. Results will be presented from an analysis of central $\mathrm{Pb}+\mathrm{Pb}$ collisions which were recorded for SPS beam energies of $20 \mathrm{~A}, 30 \mathrm{~A}, 40 \mathrm{~A}, 80 \mathrm{~A}$, and $158 \mathrm{~A} \mathrm{GeV}\left(\sqrt{S_{N N}}=6.3,7.6,8.7\right.$, 12.3 and $17.3 \mathrm{GeV})$.

\section{Onset of deconfinement}
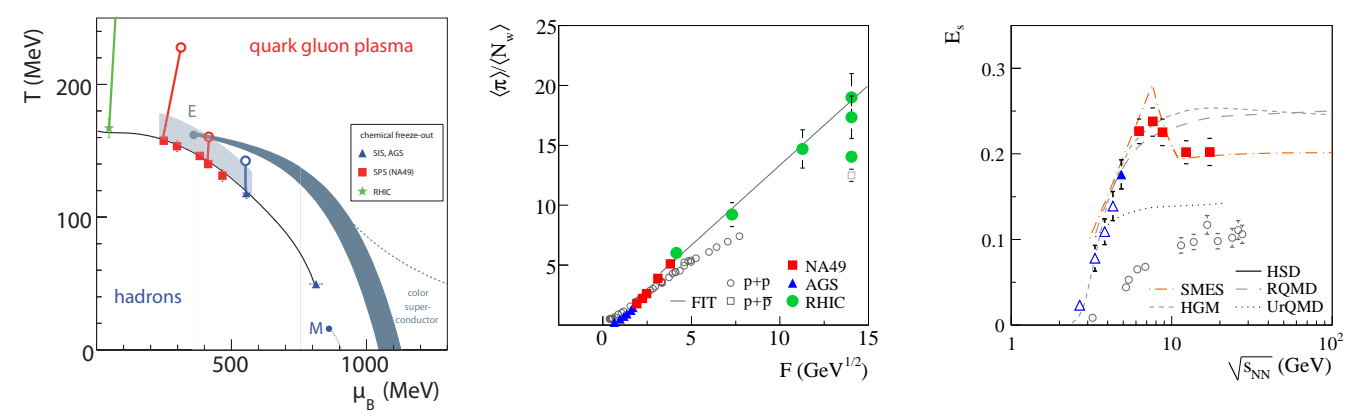

Figure 1: Left: Phase diagram of hadron matter with locations of freeze-out of the hadron composition. E denotes the estimated critical point of the first order phase boundary indicated by the shaded band. Center: Total pion yield per wounded nucleon as function of Fermi energy variable $\mathrm{F} \approx s_{N N}^{1 / 4}$. Right: Ratio $E_{S}$ of total number of strangeness carriers to pions versus collision energy. NA49 results in central $\mathrm{Pb}+\mathrm{Pb}$ collisions are shown as red squares and are compared to measurements in central $\mathrm{Au}+\mathrm{Au}$ reactions at lower and higher energies. Open circles show measurements in $\mathrm{p}+\mathrm{p}$ reactions. Curves show model predictions.

The large acceptance of the NA49 detector, its particle identification capability and the forwardbackward symmetry of $\mathrm{Pb}+\mathrm{Pb}$ reactions allow the determination of total yields of numerous particle species. The statistical hadron gas model provides a good fit to these yields with 3 parameters, namely a temperature $T$, a baryochemical potential $\mu_{B}$ and a strangeness saturation parameter $\gamma_{s}$ [5]. The resulting freeze-out points are plotted in the phase diagram of hadronic matter in 
Fig. 1 (left) and are seen to approach the estimated phase boundary and its critical end point [3]. In order to find out whether the early stage fireball actually reached hadron deconfinement the energy dependence of hadron production properties was studied in more detail. Fig. 1 (center) displays the energy dependence of the pion yield per participating nucleon. The latter is a measure of the produced entropy density in a statistical model scenario. The increase clearly steepens in the SPS energy region and can be interpreted as an increase of the effective degrees of freedom [6] by a factor $\approx 3$ [2]. This behaviour is consistent with the activation of quark-gluon degrees of freedom. The energy dependence of the production ratio of the total number of $s$ and $\bar{s}$ quarks (as deduced from strange particle yields) to pions is plotted in Fig. 1 (right). It exhibits a sharp peak at low SPS energy with a fall-off to a lower plateau value consistent with the expectation for a deconfined phase (dash-dotted curve [6]). The described features are not seen in $p+p$ collisions (open dots) nor in purely hadronic model calculations.
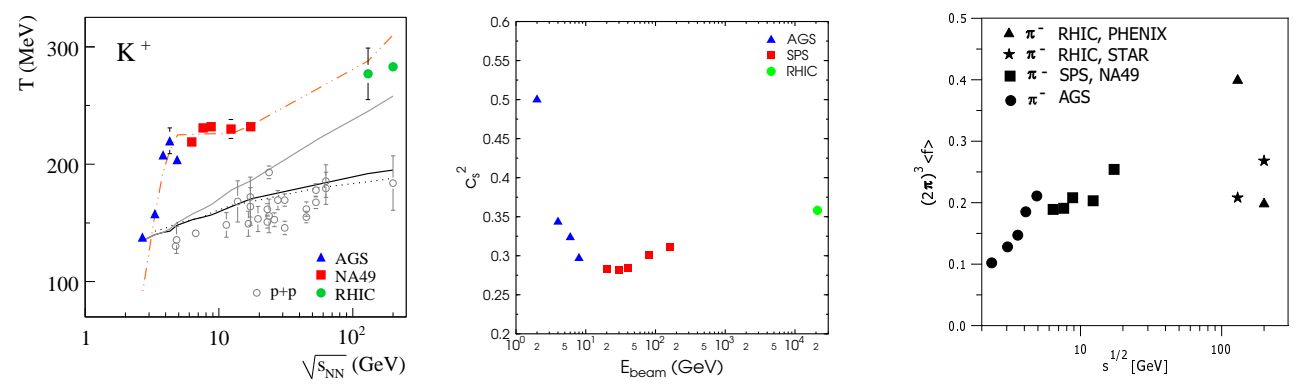

Figure 2: Left: Inverse slope parameter $T$ of the invariant transverse mass distribution of $\mathrm{K}^{+}$mesons versus collison energy. Center: Sound velocity $c_{s}$ in the fireball as determined from the width of the pion rapidity distribution using the Landau hydrodynamic model [8] versus energy. Right: Pion phase space density as determined from transverse mass spectra and fireball size derived from Bose-Einstein correlations [9]. Square symbols show NA49 results and are compared to measurements at lower and higher energies. Open circles show measurements in $\mathrm{p}+\mathrm{p}$ reactions. The curves show model predictions.

A phase transition is expected to also manifest itself in the momentum distributions and correlations of produced particles. A plot of the inverse slope parameter $T$ of the invariant transverse mass distribution of $\mathrm{K}^{+}$mesons at midrapidity is shown in Fig. 2 (left). One observes a steep rise at low energies (due to increasing radial flow in the fireball) turning into a plateau at SPS energies which is not found in $\mathrm{p}+\mathrm{p}$ reactions. This feature, which cannot be described by available hadronic models, suggests the onset of the phase transition with hadronisation through an intermediate mixed phase. A microscopic model incorporating a first order transition [7] can in fact reproduce the measurements (dash dotted curve). The softness of the equation of state near the onset of deconfinement is also seen in the sound velocity $c_{s}$ (see Fig. 2 (center)) which has been derived from the pion rapidity distributions using the Landau hydrodynamical model [8]. Finally, a step in the energy dependence of the pion phase space density (see Fig. 2 (right)), as deduced from the pion transverse mass spectra and the fireball volume estimated from Bose-Einstein correlations [9], may also be related to the onset of deconfinement. 


\section{Search for the critical point in fluctuations}
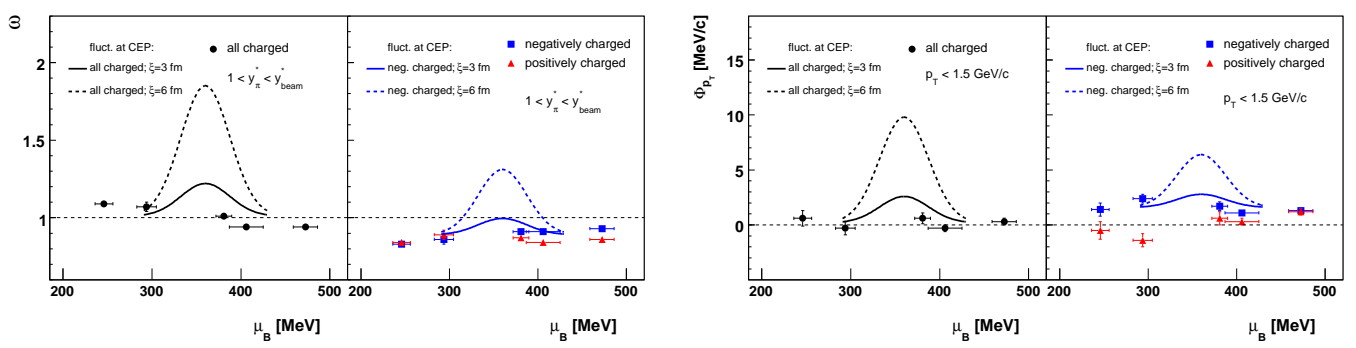

Figure 3: Left: Dependence of the scaled variance $\omega$ of the multiplicity distribution on the baryochemical potential $\mu_{B}$ for center-of-mass pion rapidities $y_{\pi}>1$. Right: Transverse momentum fluctuation measure $\Phi_{p_{T}}$ versus $\mu_{B}$ for $1.1<y_{\pi}<2$.6. Curves show expectations for the estimated critical point at $T \approx 160 \mathrm{MeV}$ and $\mu_{B} \approx 360 \mathrm{MeV}$ [3] and correlation lengths $\xi$ of 6 (dashed) and 3 (solid) fermi. The values of $\mu_{B}$ were taken from statistical hadron gas model fits to particle yields [5] at each collision energy.

The discussed energy dependence of hadron production properties showed anomalies at low SPS energies [2] which indicate that deconfinement is reached in the early stage of the produced fireball for beam energies above about $30 \mathrm{~A} \mathrm{GeV}$. Thus the evolution path of the fireball could pass close enough to the critical point such that its effect may show up as a maximum in fluctuations [10], in particular of particle multiplicity or transverse momenta. The results of the NA49 study of multiplicity [11] and transverse momentum fluctuations [12] are plotted in Fig. 3 and compared to expectations $[10,13]$ for the QCD critical point assuming the location estimated in [3]. Note that its effect is reduced both by the finite size of the fireball (correlation length $\xi$ between 3 and 6 fermi) and the limited acceptance of NA49. Unfortunately, no indication of a peak is observed in the data.
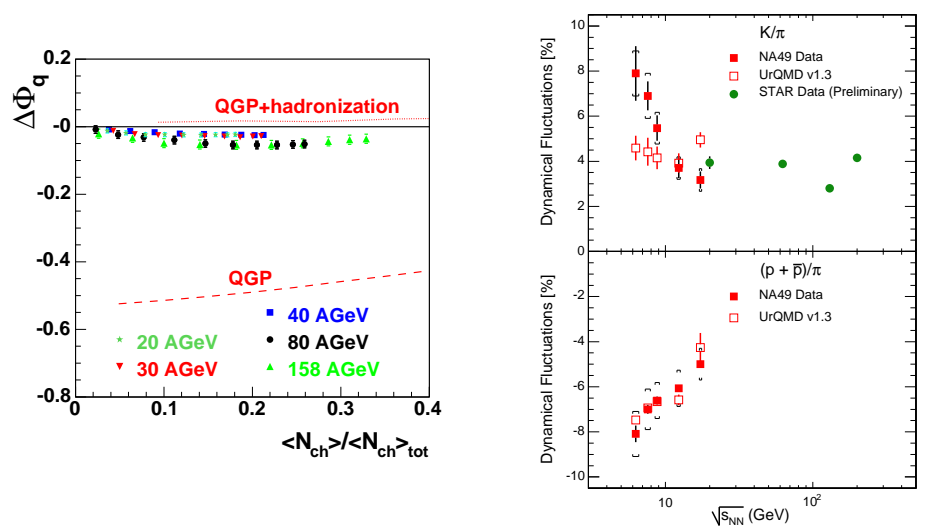

Figure 4: Left: Electric charge fluctuations at SPS energies as function of the fraction of accepted particles (rapidity window). The plotted measure $\Delta \Phi_{q}$ is corrected for global charge conservation. Right: Nonstatistical event-to-event fluctuations of the $\mathrm{K} / \pi$ and $(p+\bar{p}) / \pi$ ratios versus energy. NA49 data are shown as solid squares, predictions of the UrQMD model by open squares and results from RHIC by dots. 
NA49 also measured fluctuations of the electric charge which were speculated to be strongly reduced in a QGP [14]. No such effect was found [15] as demonstrated in Fig. 4 (left) in accordance with results from RHIC. Apparently the hadronisation and subsequent evolution of the fireball erases the effect. Finally, Fig. 4 (right) shows the non-statistical event-to-event fluctuations of the $\mathrm{K} / \pi$ and $(p+\bar{p}) / \pi$ ratios versus energy. The rise of the $\mathrm{K} / \pi$ fluctuations towards lower SPS energies is probably due to the onset of deconfinement [17] whereas the negative values for $(p+\bar{p}) / \pi$ can be understood as an effect of nucleon resonance production.

\section{Conclusion}

Measurements of NA49 at the CERN SPS indicate that deconfinement starts to occur at the early stage of central $\mathrm{Pb}+\mathrm{Pb}$ collisions for beam energies above about $30 \mathrm{~A} \mathrm{GeV}$. The freeze-out of the produced fireball happens close to the estimated position of the critical point in the phase diagram of hadron matter. However, no signals for fluctuations associated with the existence of the critical point have been found. The search for the critical point will continue at the SPS with experiment NA61 using lighter nuclei in order to possibly move the freeze-out point of the fireball closer to the critical point. Furthermore, the STAR experiment will search in Au+Au collisions with a low energy scan at RHIC with better acceptance and sensitivity. These complementary programs are expected to start in 2010/2011. Finally, there are also low-energy experiments planned at NICA in DUBNA and CBM at GSI which will join the search in later years.

\section{References}

[1] U. Heinz and M. Jacob, preprint nucl-th/0002042 (2000).

[2] C. Alt et al., Phys. Rev. C 77, 024903 (2008).

[3] Z. Fodor and S. Katz, J. High Energy Phys. 0404, 50 (2004).

[4] S. Afanasiev et al., Nucl. Instrum. Methods A 430, 210 (1999).

[5] F. Becattini et al., Phys. Rev. C 69, 024905 (2004); ibid. 73, 044905 (2006).

[6] M. Gazdzicki and M. Gorenstein, Acta Phys. Polon. B 30, 2705 (1999).

[7] S. Hama et al., Braz. J. Phys. 34, 322 (2004).

[8] H. Petersen and M. Bleicher, preprint nucl-th/0611001 (2006).

[9] S. Akkelin and Y. Sinyukov, Phys. Rev. C 73, 034908 (2006).

[10] M. Stephanov, K. Rajagopal and E. Shuryak, Phys. Rev. C 60, 114028 (1999).

[11] C. Alt et al., Phys. Rev. C 78, 034914 (2008).

[12] C. Alt et al. (NA49 collaboration), preprint arXiv:0810.5580 (2008).

[13] Y. Hatta and T. Ikeda, Phys. Rev. D 67, 014028 (2003).

[14] S. Jeon and V. Koch, Phys. Rev. Lett. 85, 2076 (2000); M. Asakawa, U. Heinz and B. Mueller, Phys. Rev. Lett. 85, 2072 (2000).

[15] C. Alt et al., Phys. Rev. C 70, 064903 (2004).

[16] C. Alt et al. (NA49 collaboration), preprint arXiv:0808.1237 (2008).

[17] M. Gorenstein, M. Gazdzicki and O. Zozula, Phys. Lett. B 585, 237 (2004). 